\title{
Theoretical properties of regularities in the oscillation spectra of A-F main-sequence stars
}

\author{
Juan Carlos Suárez ${ }^{1}$, Antonio García Hernández ${ }^{2}$, Andrés Moya ${ }^{3}$, \\ Carlos Rodrigo ${ }^{3,4}$, Enrique Solano $^{3,4}$, Rafael Garrido ${ }^{1}$, \\ and José R. Rodón ${ }^{1}$ \\ ${ }^{1}$ Instituto de Astrofísica de Andalucía (CSIC) \\ CP3004, Granada, Spain. \\ email: jcsuarez@iaa.es \\ ${ }^{2}$ Centro de Astrofísica, Universidade do Porto \\ Rua das Estrelas 4150-762, Porto, Portugal \\ email: agh@astro.up.pt \\ ${ }^{3}$ Dept. Astrofísica CAB (INTA-CSIC). ESAC Campus \\ P.O. Box 78. 28691 Villanueva de la Cañada, Madrid, Spain \\ email: amoya@cab.inta-csic.es, esm@cab.inta-csic.es, crb@cab.inta-csic.es \\ ${ }^{4}$ Spanish Virtual Observatory
}

\begin{abstract}
We study the theoretical properties of the regular spacings found in the oscillation spectra of $\delta$ Scuti stars. A linear relation between the large separation and the mean density is predicted to be found in the low-frequency domain (i.e. radial orders spanning from 1 to 8 , approximately) of the main-sequence $\delta$ Scuti stars' oscillation spectrum. This implies an independent direct measure of the average density of $\delta$ Scuti stars, analogous to that of the Sun, and places tight constraints on the mode identification and hence on the stellar internal structure and dynamics, and allows a determination the radii of planets orbiting around $\delta$ Scuti stars with unprecedented precision. This opens the way for studying the evolution of regular patterns in pulsating stars, and its relation to stellar structure and evolution.
\end{abstract}

Keywords. stars: evolution, oscillations (including pulsations), stars: interiors, stars: variables: $\delta$ Scuti.

\section{Introduction}

Thanks to great efforts made in long ground-based multi-site campaigns of A-F type pulsating stars, it was possible to find regularities in the oscillation spectra of some $\delta$ Scuti stars, e.g. for CD-24⒌ 7599 , with a regular spacing of around $26 \mu \mathrm{Hz}$ (Handler et al. 1997), or the well-studied star FG Vir, for which a regular spacing around $46 \mu \mathrm{Hz}$ (Breger et al. 2009) in its oscillation spectrum composed of 58 frequencies was found. Those works used different techniques, the histogram of frequency differences and the Fourier transform, to detect the regularities.

Later on, similar studies were performed using precise data from space, e.g. the 88 frequencies in the oscillation spectrum of the $\delta$ Scuti star HD 209775 (Matthews 2007) observed by the MOST satellite, finding a regular spacing of $\sim 50 \mu \mathrm{Hz}$. More recently, regular patterns were also found in the oscillation spectra of $\delta$ Scuti stars observed by CoRoT (García Hernández et al. 2009, Mantegazza et al. 2012, García Hernández et al. 2013) and Kepler (Hernández et al. 2013). The large number of oscillation modes and the wide frequency range cause the regular spacings to arise without making any 
Table 1. Ranges of the four parameters used to construct the current model dataset representative of intermediate-mass stars.

\begin{tabular}{c|c|c|c|}
\hline Parameter & Lowest & Highest & Step \\
\hline$M / M_{\odot}$ & 1.25 & 2.20 & 0.01 \\
{$[\mathrm{Fe} / \mathrm{H}]$} & -0.52 & +0.08 & 0.20 \\
$\alpha_{\mathrm{ML}}$ & 0.50 & 1.50 & 0.50 \\
$d_{\mathrm{ov}}$ & 0.10 & 0.30 & 0.10 \\
\hline
\end{tabular}

assumption about the distribution of the modes. Moreover, those regularities were found to be bounded (within $5 \mu \mathrm{Hz}$ ) in the very low radial-order modes, different from the so-called asymptotic regime corresponding to frequencies with radial orders above $n=$ 6 approximately (e.g. Antoci et al. 2011). The distance between these two regimes is approximately of the order of the bounded region.

This work intends to understand the physical properties of the periodicities observed in the low-frequency domain of $\delta$ Scuti stars, and also to search for any possible connection between two predicted periodicity regimes (low- and high-frequency domains).

\section{Method}

We examine a dense sample of asteroseismic models representative of A-F mainsequence stars, i.e. covering the corresponding area in the H-R diagram where classical pulsations for these stars are expected. We constructed a model collection composed of approximately $5 \times 10^{5}$ models by varying four of the main physical parameters typically used in the field for the modelling such stars (see Table 1). For the sake of homogeneity and precision of the asteroseismic mode sample, models were computed following the prescriptions suggested by the ESTA/CoRoT† working group (see Moya et al. 2008).

Pre- and post-main sequence evolutionary stages present more complex oscillation spectra, so for simplicity this work is focused on the main sequence. Although the work was initially planned to make a multivariable analysis, the first logical step is to analyse the regularities as large separations (as claimed in previous works, mainly in García Hernández et al. 2009) with respect to the mean density of stars.

\section{Rotation and mixed modes}

Rotation effects on oscillations are commonly taken into account through the perturbation approximation (Dziembowski \& Goode 1992), which is limited to slow-to-moderate rotation, i.e. small stellar deformations (e.g. Suárez et al. 2005, Reese et al. 2006). For moderate-to-rapid rotators, a complete calculation of the oscillation modes on a deformed star (Lignières et al. 2006) becomes necessary. However, nowadays this calculation is available for polytropic models and for some more realistic 2D stellar models on the ZAMS based on the self-consistent field (SCF) method (Jackson et al. 2005, MacGregor et al. 2007). Furthermore, the latter models together with the calculation of non-perturbative oscillations require a significant amount of computing resources as well as time of computation. Therefore, the use of proper modeling for rapidly rotating stars would be impractical for the present work.

$$
\dagger \text { http://www.astro.up.pt/corot/ }
$$




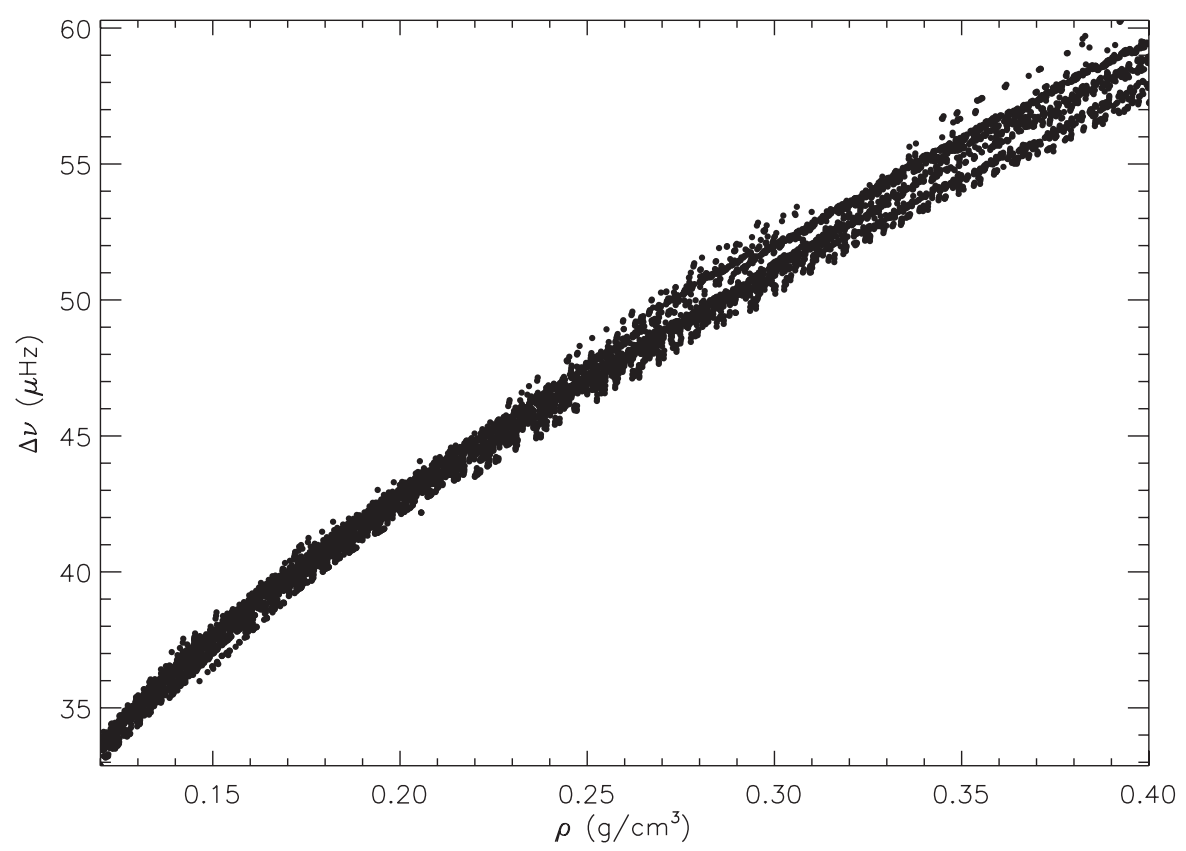

Figure 1. Theoretical large spacing, $\Delta \nu$, as a function of the mean density computed on the model grid. Time evolution is from right to left. The figure was obtained using the graphic tools of a virtual observatory service developed for this work (the tool is presented in Suárez et al. 2014).

On the other hand, since periodicities are indeed observed, it might be concluded that rotation effects are not sufficient to break the regularities presumably composed by radial modes and non-radial $m=0$ modes. Indeed, non-perturbative calculations of the oscillation spectra for rapidly rotating polytropic models indicate that, as rotation increases, the asymptotic structure of the non-rotating frequency spectrum is replaced by a new form of organization (Reese et al. 2008, 2009). This new mode-frequency organization also exhibits regular structures, including large separations (Lignières et al. 2010) whose variation from the non-rotating case is negligible (Lignières et al. 2006). Furthermore, calculations of non-perturbative oscillation frequencies on SCF models show a maximum variation of the large separation of around $2.3 \mu \mathrm{Hz}$ for stars rotating up to $40 \%$ of the Keplerian velocity (Reese, private comm.), which is small compared with the interval in which the periodicities are observed $(\sim 10 \mu \mathrm{Hz})$. Considering all the above theoretical arguments, we are allowed to use non-rotating models for the present study.

Another issue that might hamper the detection of periodicities is the presence of mixed modes. This phenomenon is implicitly considered in our work since our models cover the whole main-sequence phase. However, the phenomenon is not fully covered, since both the polytropic and the SCF models in ZAMS are not expected to properly show the avoided crossing phenomenon. More evolved SCF models should be studied in order to better analyse the impact of rotation (and the mixed) modes in the detection of regularities (work in progress).

\section{Results and discussion}

We found the following theoretical linear relation

$$
\Delta \nu / \Delta \nu_{\odot}=0.776\left(\rho / \rho_{\odot}\right)^{0.46}
$$


between the large separation (calculated in the $\delta$ Scuti lower frequency range) and the mean density obtained from the grid of models and represented in Fig. 1. This behavior is quite close to $\Delta \nu \propto \rho^{1 / 2}$ predicted for solar-like stars, opening the way for studying the evolution of regular patterns in pulsating stars, and its relation to stellar evolution.

We present no errors in the coefficients of the fitting because not all the theoretical $\left(\Delta \nu_{i}, \bar{\rho}_{i}\right)$ are independent from each other, and therefore regression error estimates are meaningless. Nevertheless it is possible to analyse the domain of validity of the method. The minimum error considered comes from the effect of rotation, i.e. $2.3 \mu \mathrm{Hz}$ corresponding to the variation of the large separation with the stellar deformation due to rotation. This yields maximum errors predicted for the estimate of the mean density range from $11 \%$ to $21 \%$ of the total variation of $\bar{\rho}$ in the main sequence (see details in Suárez et al. 2014).

The diagnostic applied to real stars (see details in Suárez et al. 2014) is consistent with previous results found in the literature, making Eq 4.1 a powerful diagnostic tool for the study of A-F stars, including $\delta$ Scuti stars and/or the hybrid phenomenon not yet understood (Uytterhoeven et al. 2011). The method also provides an estimate of the frequency of the fundamental radial mode. The strength of this diagnostic tool is that it is almost model independent, since all the models contained in the heterogenous dataset follow the same trend.

In addition, these results extend the characterization (using asteroseismology) of planetary systems to A-F type hosting stars. Most of the planets discovered by direct imaging are found to be orbiting such stars. These are critical to understand the spin-orbital interactions between the planets and the hosting star (e.g. Wright et al. 2011).

\section{References}

Antoci, V., Handler, G., Campante, T. L., et al. 2011, Nature, 477, 570

Breger, M., Lenz, P., \& Pamyatnykh, A. A. 2009, MNRAS, 396, 291

Dziembowski, W. A. \& Goode, P. R. 1992, ApJ, 394, 670

García Hernández, A., Moya, A., Michel, E., et al. 2009, A\&\&A, 506, 79

García Hernández, A., Moya, A., Michel, E., et al. 2013, A\&A, 559, A63

Hernández, A. G., Pascual-Granado, J., Grigahcène, A., et al. 2013, in: J. C. Suárez, R. Garrido, L. A. Balona, \& J. Christensen-Dalsgaard (eds.), Stellar Pulsations: Impact of New Instrumentation and New Insights, (Berlin, Heidelberg: Springer), p. 61

Handler, G., Pikall, H., O'Donoghue, D., et al. 1997, MNRAS, 286, 303

Jackson, S., MacGregor, K. B., \& Skumanich, A. 2005, ApJS, 156, 245

Lignières, F., Rieutord, M., \& Reese, D. 2006, A\& $A$, 455, 607

Lignières, F., Georgeot, B., \& Ballot, J. 2010, AN, 331, 1053

MacGregor, K. B., Jackson, S., Skumanich, A., \& Metcalfe, T. S. 2007, ApJ, 663, 560

Mantegazza, L., Poretti, E., Michel, E., et al. 2012, A\& A, 542, 24

Matthews, J. M. 2007, CoAst, 150, 333

Moya, A., Christensen-Dalsgaard, J., Charpinet, S., et al. 2008, ApछSSS, 316, 231

Reese, D., Lignières, F., \& Rieutord, M. 2006, A\&SA, 455, 621

Reese, D., Lignières, F., \& Rieutord, M. 2008, A\&SA, 481, 449

Reese, D. R., Thompson, M. J., MacGregor, K. B., et al. 2009, A\&A, 506, 183

Suárez, J. C., Bruntt, H., \& Buzasi, D. 2005, A\&A, 438, 633

Suárez, J. C., García Hernández, A., Moya, A., et al. 2014, A\&̛A, in press

Uytterhoeven, K., Moya, A., Grigahcène, A., et al. 2011, A\&A, 534, 125

Wright, D. J., Chené, A. N., De Cat, P., et al. 2011, ApJ, 728, L20 